\title{
Digital audio filter design based on YSS920B
}

\author{
Mang Zhou, a \\ ${ }^{1}$ ChongQing College of Electronic Engineering, ChongQing 401331,P. R. China \\ a zhou_mang@126.com
}

Key words: YSS920B; Digital Audio Filter; IIR Filter; Equalizer; PEQ

Abstract: This paper introduces the system design of the YSS920B digital audio DSP processing chip based on the Yamaha Corporation. The design of two order variable parameter digital equalizer is studied, and the key problems of the filter design are discussed from two aspects: system structure and variable parameters derived from the equalizer to the filter. The filter has excellent sound quality and a variety of audio data stream decoding ability, should be used for high-end AV receiver.

\section{Introduction}

For high-end audio equipment, you can use the equalizer to improve the frequency response of the amplifier and speakers. In the early stage, the equalizer is based on analog signal. With the development and popularization of DSP digital signal processing technology, the technology has been widely used in the field of audio technology. At present, digital equalization technology is mainly used in computer audio processing, media player and professional audio equipment, etc.. In this paper, the design of the two order variable parameter digital equalizer is studied. The frequency characteristic of the equalizer is analyzed by using MATLAB software. The influence of parameter variation on the performance of the equalizer is discussed.

Digital equalizer is evolved from the digital audio filter, the equalizer design is formulated for different frequency response characteristics of the filter design, but according to the theory of conventional filter design or with the aid of the MATLAB software design is a fixed frequency characteristics and parameters of the filter, users can not be real-time change the filter frequency characteristic parameters, so equalizer design is according to the requirement of the equalizer can flexibly change the frequency characteristic parameters. In this paper, the calculation method of the balanced filter can be calculated by the main index of the corresponding IIR two order filter parameters, and provide an effective method for the real-time adjustment of the equalizer parameters.

\section{Audio digital filter system structure}

The system takes the DSP chip YSS920B as the digital audio filter processor, as shown in Figure 1. The chip is designed for processing high quality audio data of the DSP processor, with 16 channels of audio data channels, 32 bit floating-point arithmetic.

Taking into account the real-time adjustment of the user filter parameters and the complex operation in order to convert the filter parameters into DSP filter transfer function parameters, a high speed microcontroller C8051F340 acts as system host MCU, with a single clock instruction cycle, the internal clock frequency of up to $50 \mathrm{MHz}$. In addition the MCU also need to control DSP processor reset, microcode downloads boot, and chip initialization settings on the A / D and D /A. 


\section{Digital equalization filter algorithm}

main parameters of bandpass filter

As shown in Figure 2, the center frequency $f_{0}$ is located in the middle of the pass band. $f_{P H}$ is

$-3 \mathrm{~dB}$ upper cutoff frequency and $f_{P L}$ is the $-3 \mathrm{~dB}$ lower cutoff frequency.

Bandwidth: $\quad \mathrm{Bw}=f_{P H}-f_{P L}$

Quality factor: $\mathrm{Q}=f_{P H}-f_{P L}$

The higher $\mathrm{Q}$ value indicates that the frequency of the filter is centered at the center frequency and the selectivity to frequency is better.

In practical application, digital equalizer with multi-stage adjustable parameters is composed by Serial connection of a plurality of two order IIR filters.
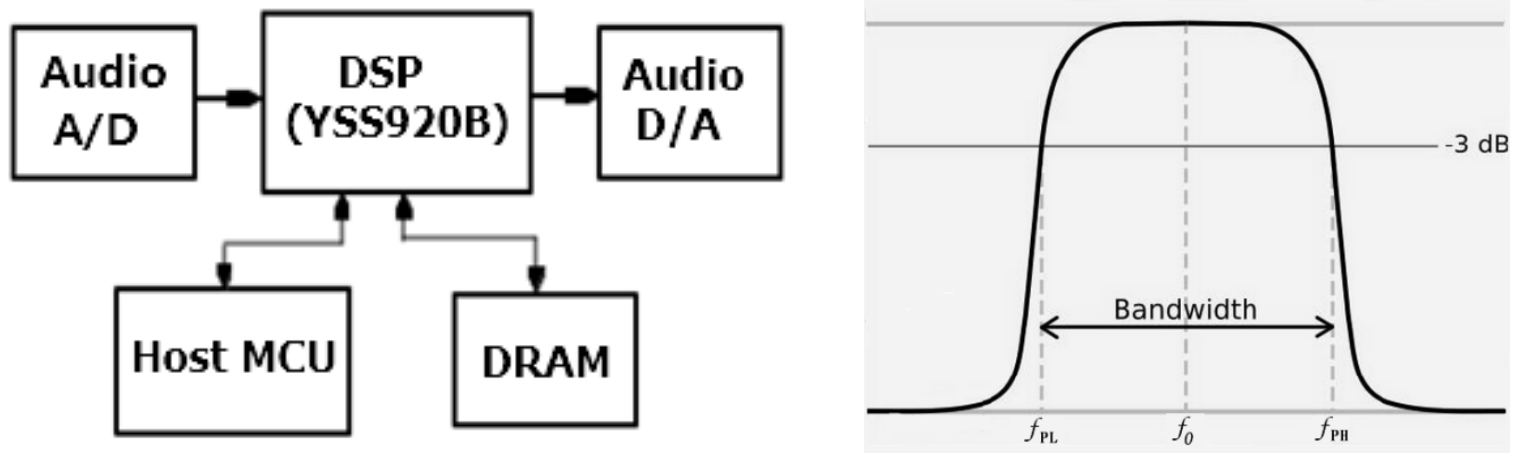

Figure 1 system structure diagram

Figure 2 frequency characteristics of band pass filter

calculation of equalization filter parameter

Taking into account the real-time operation of the DSP processor, we should try to reduce the computation of the equalizer filter algorithm, and can choose the two order IIR filter. The transfer function of the filter is as follows:

$$
H(z)=\frac{b_{0}+b_{1} z^{-1}+b_{2} z^{-2}}{a_{0}-a_{1} z^{-1}-a_{2} z^{-2}}
$$

For a two order IIR filter, the technical indicators are:

Central frequency f0 $(\mathrm{Hz})$; sampling frequency fs $(\mathrm{Hz})$; gain $\mathrm{Gn}(\mathrm{DB})$; quality factor Q.

Offen used sampling frequency fs $=48 \mathrm{kHz}$

The calculation formula of the parameters of the transfer function from Equalizer parameters is given.

For the convenience of calculation, the following values are calculated:

$$
A=\sqrt{10^{G n / 20}}, \quad \omega_{0}=2 \pi f_{0} / f_{s}, \quad \alpha=\sin \omega_{0} /(2 Q)
$$


Calculation of transfer function parameters of bandpass filter:

$$
\begin{aligned}
& b_{0}=1+\alpha \mathrm{A}, \quad b_{1}=-2 \cos \omega_{0}, \quad b_{2}=1-\alpha \mathrm{A} \\
& a_{0}=1+\alpha / \mathrm{A}, \quad a_{1}=-2 \cos \omega_{0}, \quad a_{2}=1-\alpha / \mathrm{A}
\end{aligned}
$$

Calculation of transfer function parameters for high pass filter:

$$
\begin{aligned}
& b_{0}=\left(1+\cos \omega_{0}\right) / 2, \quad b_{1}=-1-\cos \omega_{0}, \quad b_{2}=\left(1+\cos \omega_{0}\right) / 2 \\
& a_{0}=1+\alpha, \quad a_{1}=-2 \cos \omega_{0}, \quad a_{2}=1-\alpha
\end{aligned}
$$

Calculation of transfer function parameters of lowpass filter:

$$
\begin{aligned}
& b_{0}=\left(1-\cos \omega_{0}\right) / 2, \quad b_{1}=1-\cos \omega_{0}, \quad b_{2}=\left(1-\cos \omega_{0}\right) / 2 \\
& a_{0}=1+\alpha, \quad a_{1}=-2 \cos \omega_{0}, \quad a_{2}=1-\alpha
\end{aligned}
$$

The following focus on response analysis of bandpass filter. Amplitude frequency response function is calculated by $\mathrm{H}(\mathrm{z})$ :

$$
\begin{aligned}
& \mathrm{H}\left(\mathrm{e}^{i \omega}\right)=\mathrm{H}(\mathrm{z}) \mid z=e^{j \omega} \\
& =\frac{\mathrm{b}_{0}+\mathrm{b}_{1}(\cos \omega-\mathrm{j} \sin \omega)+\mathrm{b}_{2}(\cos 2 \omega-\mathrm{j} \sin 2 \omega)}{\mathrm{a}_{0}+\mathrm{a}_{1}(\cos \omega-\mathrm{j} \sin \omega)+\mathrm{a}_{2}(\cos 2 \omega-\mathrm{j} \sin 2 \omega)} \\
& x_{1}{ }^{2}=\left(b_{0}+b_{1} \cos \omega+b_{2} \cos 2 \omega\right)^{2}+\left(b_{1} \sin \omega+b_{2} \sin 2 \omega\right)^{2} \\
& x_{2}{ }^{2}=\left(a_{0}+a_{1} \cos \omega+a_{2} \cos 2 \omega\right)^{2}+\left(a_{1} \sin \omega+a_{2} \sin 2 \omega\right)^{2} \\
& H(\omega)=H\left(2 \pi f_{0} / f_{s}\right)
\end{aligned}
$$

From the above derivation, the formula for calculating the amplitude-frequency characteristic is obtained:

$$
H(\omega)=\sqrt{x_{1}^{2}+x_{2}^{2}}
$$

Summary of the application of a series of formulas above:

Firstly, according to the technical parameters (gain Gn, Q, F0, and), six coefficients (a0,b1,b2,a0, a1,a2) for IIR filter is got. These six coefficients can be sent to the DSP processor after the normalization process. The DSP processor will be in accordance with the above technical indicators to achieve band-pass filter operation. The host MCU of DSP processor is required to complete the conversion of the six coefficients from the technical indicators to the filter, and each time the user to modify the balance filter technology, the host MCU must complete the conversion 
of the parameters, then the parameters sent to the DSP processor.

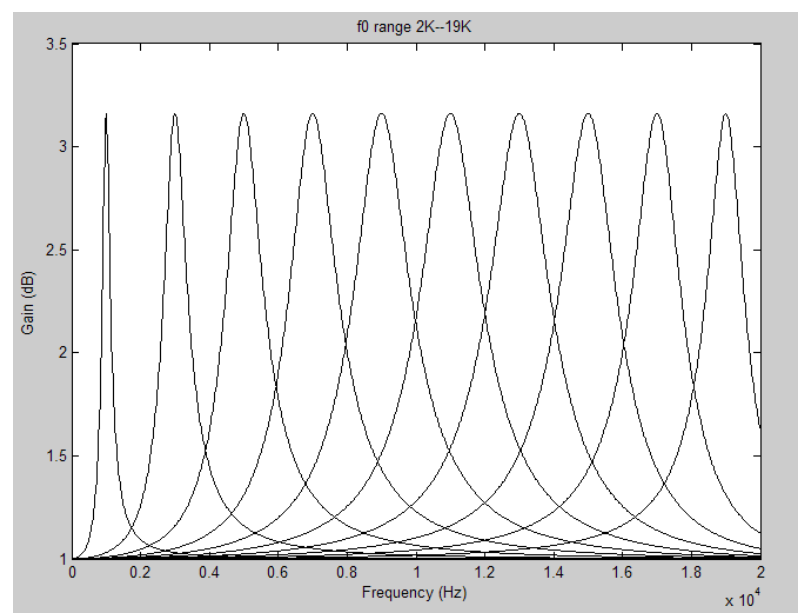

Figure 3 Full band amplitudefrequency characteristic

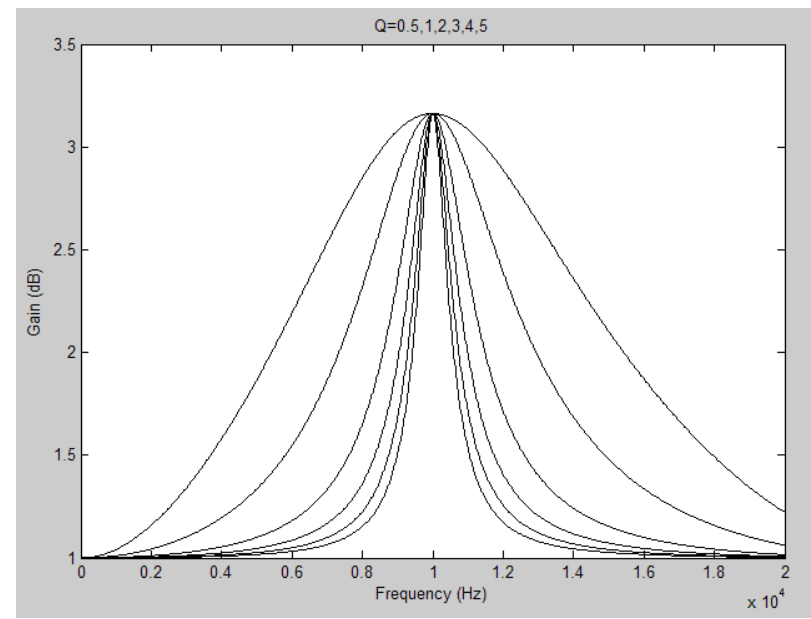

Figure 5 amplitude-frequency when Q changed

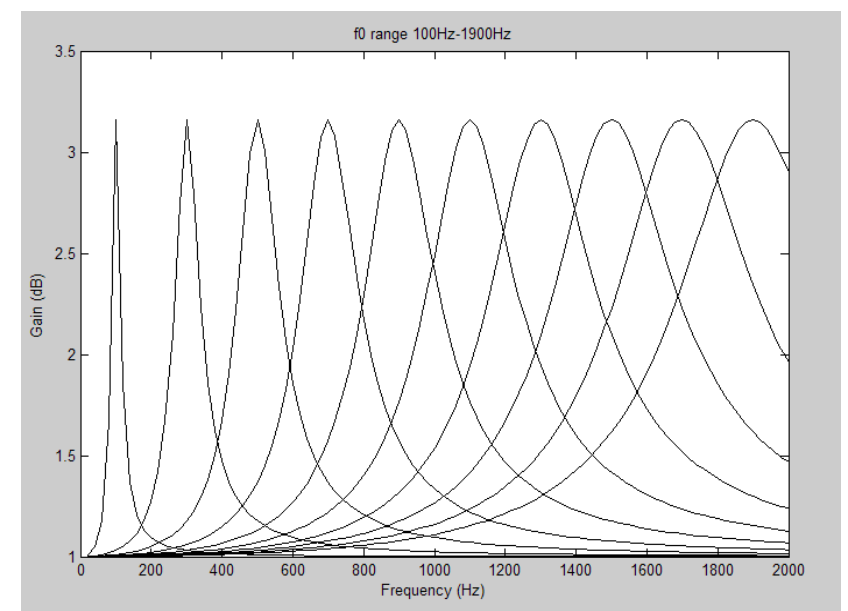

Figure 4 Low frequency band amplitude-frequency characteristic

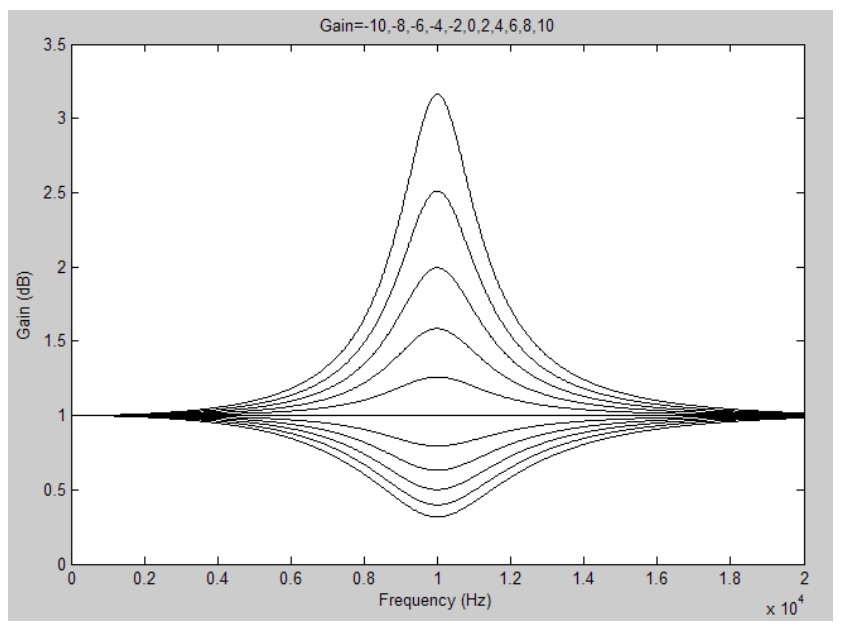

Figure 6 amplitude-frequency when Gain changed

Figure 3 to figure 6 are the filter amplitude-frequency characteristics by modify the different parameters. figure 4 and figure 3 are two frequency characteristics to adjust the center frequency f0, with the frequency of the change, the peak point of the curve will move corresponding, but the gain and $\mathrm{Q}$ values will not change. Figure 5 shows that when the $\mathrm{Q}$ value is increased, the area of the curve becomes smaller and the shape is more steep.

When using a number of two order IIR filter to work together in the same input signal, it can constitute a $20 \mathrm{~Hz} 20 \mathrm{kHz}$ full band audio equalizer. Figure 7 shows the interface of equalizer system for the application of microphone. The 10 segments equalizer is composed of 10 IIR filters, two of which are used for high and low filtering, and the other 8 IIR filters are bandpass filters. In order to achieve the filtering requirements of various audio equipment, Each IIR filter can be adjusted independently. 


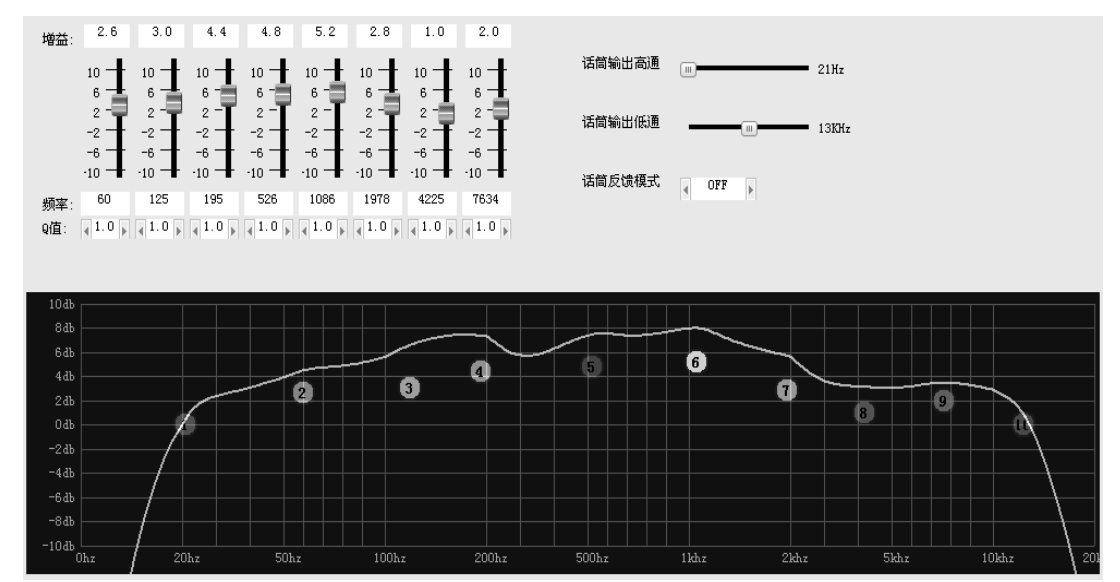

Figure 7 multi segment PEQ amplitude-frequency characteristics

\section{DSP microcode download}

DSP internal memory can be divided into 3 zones, respectively, the parameters area (RAM Coefficient), the program code area (RAM Program) and the address area (RAM Address). The download process is shown in figure 8 . The filter coefficients of digital equalizer is placed in the parameter area, and the filter coefficients can be set up in the process of downloading, and the filter parameters can be modified by the upper computer when DSP running.

\section{Conclusion}

In this paper, a digital equalization filter based on DSP chip YSS920B is introduced, the conversion method of equalizer parameters to filter coefficients is given, and the algorithm is simulated. After testing the system, it can realize the function of digital audio equalizer. Users can choose and customize various sound effects. the flexibility to set the parameters of the equalizer can meet the audio signal processing demand of frequency response of digital equalizer for the purpose to improve music sound signal. This method is simple and feasible and has been put into the product application.

\section{References}

[1]A.Oppenheim,R.Schafer and J.Buck, Discrete-Time Signal Processing, second edition Prentice-Hall,1999.

[2] J.Proakis and D.G.Manolakis, Digital Signal Processing, fourth edition Prentice-Hall, 2007.

[3] A.Antoniou, Digital Filters,McGraw-Hill Inc,NY, 1993.

[4]A.G. Deczky, Unispherical windows, Proceedings IEEE ISCS,vol.II,pp.85-89, 2001.

[5] M.Jascula. New windows family based on modified Legendre polynomials, Proceedings IEEE IMTC pp.553-556, 2002.

[6] C.M.Zierhofer, Data window with tunable side lobe ripple decay, IEEE Signal Processing Letters,vol.14, no.11,Nov. 2007.

[7]A.Papoulis Probability, Random, Variables, and Stochastic Processes, McGraw-Hill, 2002.

[8]Lawrence R.Rabiner, Linear Program Design of Finite Impulse Response Digital Filters, [J] IEEE Transactions on audio and electracoustics, 1972,20(4):280-288

[9]M.R.Arulalan,H.S.Jamadani,Ashok Rao. Novel Window Functions For Digital Filters, 
IEEETrans 2008 [C].[s.1]: Fifth International Conference on Information Technology,2008:1184-1185.

[10] Peng Shiyu a class variable parameter digital equalizer design, modern electronic technology, 2011,34 (7) 100-103

[11] However, Li Qian Zhou Nanji in the frequency domain filter design digital equalizer electronic design engineering 2011,19 (2) 162-164 